\title{
Relación entre depresión y adherencia al tratamiento en diabetes tipo 2, considerando la red de apoyo social, las estrategias de afrontamiento y el sexo
}

\section{Relationship Between Depression and Adherence to Treatment in Diabetes, Considering Social Support Networks, Coping Skills and Sex}

José Eduardo Rondón Bernard

Universidad Central de Venezuela y Universidad

Católica Andrés Bello, Venezuela
Luisa Angelucci Bastidas

Universidad Católica Andrés Bello y Universidad Simón Bolívar, Venezuela

\section{Resumen}

Desde una aproximación biopsicosocial, se pretendió determinar el modelo en diabetes que presenta un mayor ajuste a los datos: aquel donde la adherencia al tratamiento predice la depresión o aquel en que la depresión es el predictor de la adherencia. Se consideraron, en ambos, el apoyo social, las estrategias de afrontamiento y el sexo. Se utilizó un diseño transeccional-causal en 278 pacientes con un promedio de 59 años. Se empleó el Cuestionario Tridimensional de la Depresión, el Instrumento para Medir el Estilo de Vida en Diabéticos, Cuestionario de Estrategias de Afrontamiento y la Escala de Social Network and Support. Al evaluar los modelos de ecuaciones estructurales, se obtuvieron las mismas medidas de bondad de ajuste, con relaciones similares entre variables, por lo que ambos modelos poseen un ajuste aceptable. Ser mujer, poseer menor estrategia de evitación, presentar mayor red de apoyo, tener menor adherencia al tratamiento, conlleva a una menor depresión. Los resultados pueden servir de base para diseñar intervenciones psicológicas para aumentar la adherencia y evitar la depresión.

Palabras clave: diabetes, depresión, adherencia al tratamiento, estrategias de afrontamiento, apoyo social.

José Eduardo Rondón Bernard. Universidad Central de Venezuela y Universidad Católica Andrés Bello, Caracas, Venezuela; Luisa T. Angelucci Bastidas; Universidad Católica Andrés Bello y Universidad Simón Bolívar, Caracas, Venezuela.

La correspondencia en relación con este artículo se dirige a José Eduardo Rondón Bernard, Universidad Central de Venezuela (UCV) y Universidad Católica Andrés Bello (UCAB), Caracas, Venezuela. Dirección electrónica: bernard85@gmail.com 


\begin{abstract}
:
From a biopsychosocial perspective, the aim of the study was to determine which diabetes model presents the best fit to the data: one in which treatment adherence predicts depression, or one in which depression predicts treatment adherence, considering in both cases social support, coping strategies, and sex. This was assessed via a causal cross-sectional design with a sample of 278 patients, with an average age of 59 years. Measures included the Three-dimensional Depression Questionnaire, Diabetics' Lifestyle Instrument, Coping Strategies Questionnaire, and Social Network and Support Scale. Structural equation modeling showed a similar fit for indexes from both models; therefore, it can be stated that these models present an acceptable fit to the data. Being a woman, less avoidance strategy, greater support networks, and less treatment adherence, produces less depression. These results will allow researchers to design psychological interventions to increase treatment adherence, and to prevent depression.
\end{abstract}

Keywords: Diabetes, Depression, Adherence to treatment, Coping strategies, Social support.

La depresión es el desorden afectivo más frecuente en la población adulta y una de las causas más importantes de incapacidad en el mundo (Dahai et al., 2017; Wu et al., 2020). A menudo, es precedida por una enfermedad física generalmente crónica (Serrano et al., 2012); sin embargo, se plantea que la relación depresión-enfermedad crónica puede darse por dos vías: a) la depresión como causa de enfermedad por todos los cambios biológicos y comportamentales que produce y b) la depresión como consecuencia de la enfermedad, por el impacto del diagnóstico o la dificultad de asumir los cambios en el estilo de vida que se deben cumplir para recuperar la salud (Lyketsos, 2010).

Las personas con diabetes tienen doble riesgo de padecer depresión que la población general, no solo por sus complicaciones crónicas, sino también por los cambios en hábitos de salud que en ocasiones son difíciles de llevar a cabo, lo que puede empeorar su estado de salud (Caicedo et al., 2012; Domínguez \& Víamonte, 2014; Rodrígues et al., 2014; Young-Hyman et al., 2016).

La diabetes y la depresión presentan mecanismos subyacentes compartidos tanto ambientales como biológicos, tales como la activación del eje hipotalámico-hipófisis-adrenal, la inflamación, la alteración del sueño, el estilo de vida inactivo, los hábitos dietéticos deficientes y los factores de riesgo ambientales y culturales (Holt et al., 2014; Pouwer et al., 2020). Una explicación de que el padecimiento de la diabetes aumenta la probabilidad de presentar depresión es por los cambios fisiológicos, individuales, familiares y sociales que se presentan en la persona que la padece.

La exclusión o la prohibición de muchas actividades de la vida cotidiana, por personas con diabetes, origina que la depresión se presente en este tipo de paciente con mayor frecuencia que en las personas sin diabetes (Castillo-Quan et al., 2010; Serrano et al., 2012). La diabetes implica la carga emocional de una enfermedad crónica; por ende, es necesario que se tomen medidas terapéuticas tales como el cumplimiento estricto del tratamiento con los cambios que esto implica tanto para el paciente diagnosticado con esta como para el resto de los miembros de la familia (Álvarez et al., 2014).

De acuerdo con algunos autores, la depresión puede ser un factor detonador de la hiperglucemia, una barrera para la adherencia al tratamiento o ser consecuencia del impacto de la condición (Castillo \& Aguilar, 2011; Castillo-Quan et al., 2010; Holt et al., 2014; Kaltman et al., 2016). Las personas 
RELACIÓN ENTRE DEPRESIÓN Y ADHERENCIA AL TRATAMIENTO EN DIABETES TIPO 2

diagnosticadas con diabetes experimentan un proceso de ajuste que puede originar reacciones y sentimientos tales como coraje, depresión, ansiedad, frustración y pérdida de valor por la vida, lo que conlleva a una diabetes descontrolada; es decir, pacientes que no se adhieren al tratamiento (Escobedo et al., 2007; Rivas-Acuña et al., 2011).

En este sentido, Castillo y Aguilar (2011) plantean que la depresión y la adherencia al tratamiento se vinculan de diferentes maneras: en primera instancia, se puede establecer que la depresión representa una barrera terapéutica para un adecuado control metabólico. En segunda instancia, se puede plantear que la depresión es el resultado del impacto del tratamiento al requerir un cambio drástico de estilo de vida (Benítez et al., 2011; Escobedo et al., 2007; González et al., 2008).

Se ha encontrado que los síntomas y los trastornos depresivos están significativamente asociados con el inadecuado control glucémico, mayor gravedad de las complicaciones de la diabetes, menor adherencia al autocuidado de la condición, disminución de la calidad de vida, mayor discapacidad funcional y mortalidad precoz (De Groot et al., 2016; Rivas-Acuña et al., 2011; Westby et al., 2020; Wu et al., 2020). No obstante, otros autores señalan que la adherencia al tratamiento puede causar depresión, puesto que el seguimiento del tratamiento por su complejidad puede ser aversivo para el paciente al afectar más a la persona con diabetes tipo 2 (Colunga et al., 2008; Franciosi et al., 2001; Frojan \& Rubio, 2004; MassanoCardoso et al, 2020).

Existen pocos estudios para determinar la naturaleza de la relación de causalidad entre depresión y adherencia al tratamiento y algunos de ellos reportan resultados inconsistentes y de baja rigurosidad a nivel metodológico (Escobedo et al., 2007; Rivas-Acuña et al., 2011). Por lo expuesto, la presente investigación tratar de evaluar cuál sería el modelo más adecuado, aquel donde la depresión influye negativamente a la adherencia o la adherencia al tratamiento influye negativamente a la depresión (Moral de la Rubia \& Cerda, 2015; Shrestha et al., 2020).

Existen otros factores que se encuentran estrechamente vinculados a la relación entre depresión y adherencia al tratamiento, tales como el sexo, las estrategias de afrontamiento y la red de apoyo social de las personas con diabetes. Estudios recientes sostienen que las mujeres con diabetes son más propensas a presentar depresión en comparación con los hombres diagnosticados con diabetes (Álvarez et al., 2014; Benítez et al, 2011; Colunga et al, 2008; Parildar et al., 2015; Rahimi et al., 2020; Rivas-Acuña et al, 2011). Además, tienden a emplear más las estrategias de afrontamiento centradas en la emoción tales como la resignación y el aislamiento en comparación con los hombres con diabetes (Gafyels \& Wandell, 2005).

Por su parte, Salcedo-Rocha et al. (2008) plantean que las mujeres con diabetes participan, en menor medida, en actividades recreativas en comparación con los hombres; por ende, son más propensas a presentar estados emocionales depresivos. En este sentido, se expresa que el uso de estrategias de afrontamiento inadecuadas; por ejemplo, el mayor empleo de estrategias de evitación en el manejo de la condición incrementa la probabilidad de desarrollar un cuadro depresivo (Peralta, 2007; Rogers et al., 2005).

Kraaij y Gamefski (2015) señalan que el estilo de afrontamiento activo o conductual no se encuentra relacionado con la sintomatología depresiva, solo las estrategias de afrontamiento cognoscitivas tales como la rumiación, el culpar a otros y la catastrofización, de manera positiva y la reevaluación positiva y el ajuste a metas de forma inversa. Otros autores como Burns et al. (2016) enfatizan en que son las 
estrategias dirigidas a la emoción las que se encuentran relacionadas con la depresión.

Según Parildar etal.(2015), en el caso de la diabetes, un mayor tiempo con la condición, ser mujer, emplear estrategias de evitación al problema, mantener poca adherencia al tratamiento y el bajo nivel educativo se encuentran asociados con mayor posibilidad de desarrollar depresión durante el curso de la condición.

Por su parte, autores como Arteaga et al. (2017) y Rondón y Lugli (2013) plantean que el soporte social representa un factor protector de la salud en la diabetes, puesto que permite mayor calidad de vida y evita reacciones negativas como la depresión. De esta manera, el apoyo social se relaciona con la compensación metabólica de las personas con diabetes. Por tal razón, es indispensable involucrar al entorno que rodea al paciente, en cada una de las etapas de la condición, y que ello conlleve a un mejor afrontamiento y control de (Alcaíno et al., 2014; Beverly et al., 2021).

Sin embargo, en ocasiones, el apoyo puede representar un obstáculo al ajuste a la condición, pues podría fomentar la dependencia y generar miedo, frustración, rechazo y angustia y manifestarse todas en los trastornos depresivos (Hoyos et al., 2011; Karlsen et al., 2012; Montiel-Carbajal \& DomínguezGuedea, 2011). Por ejemplo, Karlsen et al. (2012) señalan que cuando existen por parte de las redes sociales "críticas", "regaños, "señalamientos" hacia las conductas inadecuadas de las personas con diabetes, aumenta la posibilidad de distrés en estos y mayor probabilidad de depresión.

Resulta importante esclarecer la relación existente entre depresión y adherencia al tratamiento al considerar el sexo, las estrategias de afrontamiento y la red de apoyo social como factores influyentes. Dichas variables, a su vez, se relacionan entre ellas. De esta forma, la presente investigación pretende determinar el modelo en diabetes tipo 2, que presenta un mayor ajuste: a) aquel donde la adherencia al tratamiento predice la depresión o b) aquel en que la depresión predice la adherencia al tratamiento.

Se plantean así dos modelos. En el modelo A, la adherencia predice la depresión y supone que: Sobre depresión:

- Los hombres diagnosticados con diabetes tipo 2 tienen mayor depresión.

- Menor empleo de estrategias de evitación, menor depresión.

- Mayor empleo de estrategias de aproximación, menor depresión.

- Mayor red de apoyo social, menor depresión.

- Mayor adherencia al tratamiento menor depresión.

Sobre adherencia al tratamiento:

- Los hombres diagnosticados con diabetes tipo 2 evidencian mayor adherencia al tratamiento.

- Mayor empleo de estrategias de evitación, menor adherencia al tratamiento.

- Menor empleo de estrategias de aproximación, menor adherencia al tratamiento

- Menor red de apoyo social, menor adherencia al tratamiento.

- Mayor depresión, menor adherencia al tratamiento. 
RELACIÓN ENTRE DEPRESIÓN Y ADHERENCIA AL TRATAMIENTO EN DIABETES TIPO 2

\section{Sobre el apoyo social:}

- Los hombres diagnosticados con diabetes tipo 2 tienen mayor red de apoyo social.

- Menor empleo de estrategias de evitación, mayor red de apoyo social.

- Mayor empleo de estrategias de aproximación, mayor red de apoyo social.

Sobre estrategias de aproximación y evitación:

- Los hombres con diagnóstico de diabetes tipo 2 tienen menor estrategia de evitación y mayor estrategia de aproximación.

En el modelo B, la depresión predice la adherencia al tratamiento. Aquí, se supone que se mantienen la misma naturaleza de todas las relaciones planteadas; sin embargo, las medidas de calidad de ajuste cambiarían al suponer que el modelo A es superior.

\section{Método}

\section{Participantes}

En el estudio participaron 278 pacientes entre hombres y mujeres con diagnóstico de diabetes tipo 2 (119 hombres, 159 mujeres), quienes asistían a una fundación que apoya a pacientes con diabetes en Venezuela, con rango entre 19 y 77 años (58.59 años \pm 11.59$)$, con promedio relativamente adecuado de hemoglobina glícosilada $(7.74 \mathrm{Hgl} \pm 2.05)$. Se empleó un tipo de muestro no probabilístico de tipo propositivo, pues se seleccionaron, intencionalmente, los sujetos de acuerdo con los siguientes criterios:

- Mínimo 1 año con diagnóstico de diabetes tipo 2.

- Sin comorbilidad con nefropatía diabética, retinopatía diabética, amputaciones, VIH o cáncer.

- Sin diagnóstico psiquiátrico a excepción de depresión.

- Que sepan leer y escribir con un mínimo de comprensión lectora.

Toda persona que no cumpliera con los anteriores criterios y que no llenaron los cuestionarios en su totalidad, se excluyeron del estudio. La participación fue voluntaria con consentimiento informado y siguiendo los lineamientos éticos de la investigación en Venezuela según la Ley de Ejercicio de la Psicología (Congreso de la República de Venezuela, 1978). Cabe resaltar que la fundación en la cual se realizó el estudio revisó previamente los cuestionarios y el procedimiento para garantizar protección de los pacientes.

A continuación, en la tabla 1 se presentan detalladamente las características de la muestra en estudio. Se consideraron el sexo, la edad, el tiempo con la condición y el estado civil. 
Tabla 1

Descripción de la muestra de personas con diabetes tipo 2

\begin{tabular}{|c|c|c|}
\hline & $\mathrm{N}$ & $\%$ \\
\hline \multicolumn{3}{|l|}{ Sexo } \\
\hline Hombres & 119 & $42.81 \%$ \\
\hline Mujeres & 159 & $57.19 \%$ \\
\hline \multicolumn{3}{|l|}{ Edad } \\
\hline 19-30 años & 10 & $3.60 \%$ \\
\hline 31-40 años & 16 & $5.80 \%$ \\
\hline 41-50 años & 30 & $10.70 \%$ \\
\hline 51-60 años & 87 & $31.30 \%$ \\
\hline $61-70$ años & 102 & $36.70 \%$ \\
\hline 71- 77 años & 33 & $11.90 \%$ \\
\hline \multicolumn{3}{|c|}{ Tiempo con diabetes } \\
\hline 1- 5 años & 110 & $39.60 \%$ \\
\hline 6- 10 años & 75 & $26.90 \%$ \\
\hline 11- 15 años & 38 & $13.70 \%$ \\
\hline 16- 20 años & 26 & $9.40 \%$ \\
\hline 21- 25 años & 10 & $3.60 \%$ \\
\hline 26- 30 años & 9 & $3.20 \%$ \\
\hline 31- 35 años & 3 & $1.10 \%$ \\
\hline 36- 40 años & 3 & $1.10 \%$ \\
\hline 41- 45 años & 1 & $0.36 \%$ \\
\hline 46- 50 años & 3 & $1.10 \%$ \\
\hline \multicolumn{3}{|l|}{ Estado civil } \\
\hline Soltero & 75 & $27.00 \%$ \\
\hline Casado & 133 & $47.80 \%$ \\
\hline Viudo & 26 & $9.40 \%$ \\
\hline Concubinato & 20 & $7.20 \%$ \\
\hline Divorciado & 24 & $8.60 \%$ \\
\hline
\end{tabular}


RELACIÓN ENTRE DEPRESIÓN Y ADHERENCIA AL TRATAMIENTO EN DIABETES TIPO 2

\section{Diseño}

De acuerdo con Montero y León (2007) es un diseño prospectivo con más de un eslabón causal, lo que permite verificar relaciones causales entre variables, donde una variable puede asumir el papel de causa, pero, a la vez, de efecto de otras variables. Se usa un modelo de ecuaciones estructurales (MEE), que permite ver efectos directos o indirectos de una variable sobre otra a través de la integración de ecuaciones lineales, donde algunas o todas las variables son latentes. En este sentido, implica un modelo de medida y un modelo de relación (Ortiz \& Fernández-Pera, 2018).

\section{Variables}

\section{Variables endógenas}

Depresión. Definición conceptual: Es un trastorno multifactorial que implica un conjunto de síntomas específicos conductuales o motores (agitación o enlentecimiento motor, intentos de suicidio, inhibición general, llanto, lenguaje escaso, adicciones, signos faciales de desesperanza y tristeza, autoagresión, verbalizaciones negativas y quejas, desarreglo personal) cognoscitivos (valoración negativa de sí mismo, del entorno y del futuro) sociales (aumento de la dependencia de otros, evitación de interacción social recreativa) y biológicos (aumento o disminución de peso, insomnio o hipersomnia, fatiga, pérdida de energía y de apetito, alteración del funcionamiento sexual, molestias corporales difusas, astenia, dolores musculares y articulares, cansancio), que ocasiona que el sujeto pierda reforzamiento de su entorno y, por ende, existan dificultades en su funcionamiento cotidiano (Jiménez, 2002; Reyes, 2013; Serrano et al., 2012; Toro-Tobar et al., 2016; Vallejo, 2011).

Definición operacional: Sumatoria de la puntuación obtenida en cada dimensión (cognitivo, fisiológico y motor) del Cuestionario Tridimensional para la Depresión de Jiménez y Miguel-Tobal (2003). Se empleó la adaptación venezolana en pacientes con enfermedades crónicas realizada por Cardozo, Guarino y Rondón (2016), la cual consta de 32 ítems, 11 en la escala motor, 12 en el cognitivo y 9 en el fisiológico. Donde a mayor puntaje total, mayor severidad de depresión.

Adhesión al tratamiento. Definición conceptual: Es la adquisición y el mantenimiento de un estilo de vida caracterizado por la administración de los hipoglicemiantes (orales o insulina) en la dosis y la frecuencia acordada con el especialista al monitoreo del nivel de glucosa en sangre, el aumento del ejercicio físico, el control emocional en las situaciones estresantes, al cumplimiento de las revisiones médicas regulares y a la realización de una dieta ajustada a sus requerimientos nutricionales, que contribuya al logro de un buen control glicémico, a la postergación de sus complicaciones y, por último, a una mejora en su calidad de vida (Rondón, 2018).

Definición operacional: Puntaje en cada una de las dimensiones (nutrición, actividad física, consumo de tabaco, consumo de alcohol, información sobre diabetes y adherencia terapéutica) del Instrumento para Medir el Estilo de Vida en Diabéticos IMEVID de López et al. (2003). Compuesto por 21 ítems. A mayor puntaje, mayor adhesión al tratamiento reflejada en cada dimensión.

Red de apoyo social. Definición conceptual: Conjunto de personas con las que se comunica un individuo, así como las características de los lazos que se establecen y los tipos de interacciones que se originan entre ellos (Otero et al., 2006). 
Definición operacional: Puntaje total obtenido en la Escala de Social Network and Support de Unden y Orth-Gomer (1989, cit. en Feldman, 2002) modificada por Feldman (2002) para la población venezolana y validado en esta población por Rondón y Lugli (2010). Constituido por 5 ítems, donde a mayor puntaje mayor red de apoyo social.

Estrategias de afrontamiento. Definición conceptual: "Son aquellos esfuerzos cognoscitivos y conductuales constantemente cambiantes que se desarrollan para manejar las demandas específicas externas y/o internas que son evaluadas como excedentes o desbordantes de los recursos del individuo" (Lazarus \& Folkman, 1986, p.164). De acuerdo con Rodríguez-Marín et al. (1993), se divide en estrategias de aproximación al problema y estrategias de evitación al problema.

Estrategias de afrontamiento de aproximación. Definición conceptual: son todos aquellos esfuerzos cognoscitivos y conductuales que aproximan al problema que está generando el estrés o a las emociones negativas concominantes (Rodríguez-Marín et al., 1993).

Definición operacional: Puntaje total en la dimensión estrategias de afrontamiento de aproximación al problema (sumatoria de las subdimensiones búsqueda de apoyo social, pensamiento positivo, planificación y búsqueda de soluciones) del Cuestionario de Estrategias de Afrontamiento de RodríguezMarín et al. (1993) adaptado por Zaragoza (2006). La dimensión de estrategias de afrontamiento de aproximación al problema se encuentra constituida por 18 ítems. El puntaje mínimo es de 18 puntos y el máximo es de 90 puntos. A mayor puntaje, mayor uso de dicha estrategia.

Estrategias de afrontamiento de evitación. Definición conceptual: Son todos aquellos esfuerzos cognoscitivos y conductuales que implican escape o huida al problema que genera estrés o a las emociones negativas concomitantes (Rodríguez-Marín et al., 1993).

Definición operacional: Puntaje total en la dimensión de estrategias de afrontamiento de evitación al problema (sumatoria de las subdimensiones de evitación, pensamiento desiderativo, culpar a otros y resignación) del Cuestionario de Estrategias de Afrontamiento de Rodríguez-Marín et al. (1993) adaptado por Zaragoza (2006). La dimensión de estrategias de afrontamiento de evitación al problema se encuentra constituida por 23 ítems. El puntaje mínimo es de 23 puntos y el máximo es de 115 puntos. A mayor puntaje mayor uso de dicha estrategia.

\section{Variable exógena}

Sexo. Definición conceptual: Hecho biológico de ser hombre o mujer (Stolcke, 2000). Definición operacional: Reporte de "hombre (H)" o "mujer (M)" en el apartado de sexo en datos sociodemográficos.

\section{Instrumentos}

Cuestionario Tridimensional para la depresión (CTD). Desarrollado por Jiménez (2002) para estudiar la depresión desde el modelo teórico de los tres sistemas de respuesta (cognitivo, fisiológico y motor). En la presente investigación se usó la adaptación venezolana en pacientes con enfermedades crónicas realizada por Cardozo et al. (2016), la cual se encuentra constituida por 32 ítems, 11 en la escala motor, 12 en el cognitivo y 9 en el fisiológico. Los 32 ítems se encuentran precedidos por el ítem de carácter criterial "me siento triste". La escala de respuesta oscila del 0 al 4 donde 0 es "casi nunca" y 4 es 
RELACIÓN ENTRE DEPRESIÓN Y ADHERENCIA AL TRATAMIENTO EN DIABETES TIPO 2

“casi siempre”. El puntaje mínimo es 0 y el máximo es 128. A mayor puntaje, mayor nivel de depresión. Los índices de confiabilidad en todas las escalas según Cardozo et al. (2016) son superiores a .75. El índice de consistencia interna de la prueba total es de .85. Las correlaciones entre escalas oscilan entre .37 y .49 y las correlaciones entre las tres escalas y la prueba total, se encuentran entre .70 y .81 . Respecto a la validez de constructo, los análisis demuestran que el cuestionario presenta una estructura factorial que explica un $31.94 \%$ de la varianza total (Cardozo et al., 2016). Para la presente investigación, los índices de consistencia interna por el alfa de Cronbach fueron: factor Motor .76, cognitivo $.75 \mathrm{y}$ fisiológico .76 .

Instrumento para Medir el Estilo de Vida en Diabéticos (IMEVID). Elaborado por López et al. (2003) instrumento de autoadministración que se emplea para medir la adherencia al tratamiento en pacientes ambulatorios con Diabetes mellitus tipo 2. Se encuentra constituido por 25 ítems que se distribuyen en siete dimensiones, a saber: Nutrición (9 ítems con un $\alpha$ de Cronbach de .61), actividad física (3 ítems con un $\alpha$ de Cronbach de .51), consumo de tabaco (2 ítems con un $\alpha$ de Cronbach de .96), consumo de alcohol (2 ítems con un $\alpha$ de Cronbach de .90), información sobre diabetes ( 2 ítems con un $\alpha$ de Cronbach de .62), emociones (3 ítems con un $\alpha$ de Cronbach de .73) y adherencia terapéutica (4 ítems con un $\alpha$ de Cronbach de .73). La corrección consiste en asignar calificaciones de 0,2 y 4 a las tres opciones de respuesta y corresponde el valor más alto para la conducta deseable. El $\alpha$ de Cronbach total es de .81, con una varianza total explicada de $60.4 \%$ (López et al., 2003). Para la presente investigación, se decidió eliminar el ítem 4 ¿cuántas tortillas come al día?, por no ajustarse a la cultura venezolana. Además, no se empleó el puntaje total de los ítems correspondientes a la dimensión "emoción" por no responder a la definición realizada del constructo, teniendo así la versión final 21 ítems y quedaron 6 dimensiones. En el presente estudio, los índices de consistencia interna por el alfa de Cronbach fueron; Nutrición .48, actividad física .22, consumo de tabaco .97, consumo de alcohol .92, información .29 y adherencia terapéutica .72 .

Escala de Integración Social (ISSI). Versión original condensada del Interview Schedule for Social Interaction de Henderson et al. de 1980 (Feldman, 2002). Su propósito es evaluar la red de apoyo social en su dimensión de integración social. La escala empleada fue la traducida al español en 2002 por Feldman. Esta versión consta de 5 ítems, cada uno con 6 opciones de respuesta (ninguna, 1-2, 3-5, 6-10, 11-15 y más de 15). El puntaje mínimo es de 5 puntos y el máximo es de 30 puntos. A mayor puntaje, mayor red de apoyo social dada por la cantidad de personas que constituyen la fuente de apoyo social, arrojando un $\alpha$ de Cronbach de .76; y una alta validez predictiva de enfermedades cardiovasculares y depresión. (Feldman, 2002). Rondón y Lugli (2010) establecen que el cuestionario en población venezolana con enfermedades crónicas posee un $\alpha$ de Cronbach de .79 y una estructura unifactorial, que explica el $55.25 \%$ de la varianza total. Para la presente investigación, el índice alfa de Cronbach fue de .68.

Cuestionario de Estrategias de Afrontamiento (CEA). Diseñado por Rodríguez-Marín et al. en 1993. Para la presente investigación se empleó la adaptación realizada por Zaragoza en el 2006. El cuestionario se encuentra constituido por 41 ítems que evalúan siete estrategias de afrontamiento las cuales son las siguientes: evitación ( 7 ítems con un $\alpha$ de Cronbach de .88), pensamiento desiderativo (5 ítems, con un $\alpha$ de Cronbach de .88), culpabilización de otros (6 ítems, con un $\alpha$ de Cronbach de .80), resignación ( 5 ítems, con un $\alpha$ de Cronbach de .88), búsqueda de apoyo social ( 8 ítems, con un $\alpha$ de Cronbach de .88), pensamiento positivo (5 ítems, con un $\alpha$ de Cronbach de .88) y planificación y búsqueda de 
soluciones (5 ítems, con un $\alpha$ de Cronbach de .80). La sumatoria de las dimensiones "búsqueda de apoyo social", "pensamiento positivo" y "planificación y búsqueda de soluciones" conforma las estrategias de aproximación al problema; y la sumatoria de las dimensiones "evasión", "pensamiento desiderativo", "culpabilización de otros" y "resignación" conforma las estrategias de evitación del problema. Se puntúan en una escala tipo likert donde 1 significa "nunca"; 2 , "rara vez"; 3, "algunas veces"; 4, "a menudo" y 5, "la mayoría de las veces". En la validación del instrumento realizada por Zaragoza (2006) para la población venezolana, el cuestionario mostró una estructura factorial cónsona con la teoría donde se explicó el $66.12 \%$ de la varianza total. A mayor puntaje, mayor uso de cada tipo de estrategia (aproximación o evitación). Para la presente investigación, los índices de consistencia interna por el alfa de Cronbach fueron de .84 para estrategias de aproximación y .83 para las de evitación.

Hoja de datos de identificación. Formato previamente diseñado, donde se le solicitó por escrito a cada uno de los participantes la siguiente información: edad, sexo, peso, tiempo con la condición, tipo de tratamiento, estado civil, tipo de religión y nivel de instrucción.

Hoja de consentimiento informado. Formato previamente diseñado por medio del cual se le informó a los participantes el propósito de la investigación y los principios éticos garantizados: riesgo del participante, libertad de coerción y confidencialidad; en este sentido, se les informó que sus datos eran anónimos y si durante el proceso de evaluación se originaba algún malestar/incomodidad podía dar fin a la misma sin tener alguna repercusión personal.

\section{Procedimiento}

Se redactó la solicitud de permiso a las autoridades de la fundación antidiabética para utilizar sus instalaciones y solicitar a los pacientes su consentimiento a participar en la fase de aplicación de la investigación. Luego, se entrenó al personal de recolección de datos en técnicas de entrevista y aplicación de los cuestionarios. Además, se le otorgó por escrito los criterios de inclusión de la muestra para que procedieran a seleccionar los participantes. Una vez seleccionado el paciente, se procedió a indicarle el propósito de la investigación y a solicitarle su consentimiento de participación por escrito. Así, se cumplió con los parámetros éticos de la investigación. Después, se siguió con la aplicación de los cuestionarios en forma de entrevista. El orden era CTD, ISSI, CEA, IMEVID. La duración promedio de aplicación fue de 30 minutos. Completada la recolección de datos, se realizó su codificación en el paquete estadístico AMOS con el propósito de ejecutar los análisis estadísticos pertinentes para responder a los objetivos del estudio y seguidamente discutir los resultados obtenidos.

\section{Análisis de datos}

Con el propósito de describir el comportamiento de las variables en estudio, se calcularon los estadísticos descriptivos de tendencia central (media), de dispersión (desviación típica, mínimo y máximo) y de las características de la distribución (asimetría y curtosis) de las variables en estudio.

Luego, se evaluaron los supuestos requeridos para realizar el MEE (normalidad de las variables, independencia de los errores, homocedasticidad de la varianza, linealidad y baja multicolinealidad entre las variables). Se ejecutaron los dos modelos: el primero donde la adherencia al tratamiento predice la depresión y el segundo donde se propone la relación contraria al considerar en ambos modelos la red 
RELACIÓN ENTRE DEPRESIÓN Y ADHERENCIA AL TRATAMIENTO EN DIABETES TIPO 2

de apoyo social, las estrategias de afrontamiento y el sexo. Para cada modelo, se obtuvo los índices de ajuste: Chi cuadrado del modelo estimado $\left(\chi^{2}\right)$, índice de bondad de ajuste (GFI), el error de aproximación cuadrático medio (RMSEA), índice de bondad de ajuste ajustado (AGFI), índice de ajuste normado de parsimonia (PNFI) e índice de ajuste comparativo (CFI); el modelo de relaciones compuesto por los coeficientes betas para cada una de las variables predictoras involucradas en el estudio y, el modelo de medida conformado por las cargas factoriales y la varianza de error de cada variable estudiada (Hair et al., 1999; Iglesias \& Sulé, 2003).

\section{Resultados}

\section{Estadísticos descriptivos}

A continuación, se presentan en la tabla 2 los estadísticos descriptivos de las dimensiones de cada variable del estudio.

Tabla 2

Estadísticos descriptivos de las dimensiones de las variables estudiadas en la muestra

\begin{tabular}{llcccccc}
\hline Variable & Dimensiones & Mínimo & Máximo & Media & DE & Asimetría & Curtosis \\
\hline Depresión & Motor & 0 & 41 & 11.60 & 8.21 & 0.99 & 0.79 \\
& Cognitivo & 0 & 40 & 5.22 & 6.47 & 2.48 & 7.73 \\
& Fisiológico & 0 & 33 & 11.52 & 7.46 & 0.57 & -0.36 \\
Adherencia al & Nutrición & 8 & 24 & 19.06 & 2.34 & -0.79 & 1.58 \\
tratamiento & Actividad física & 3 & 9 & 6.31 & 1.66 & -0.23 & -0.80 \\
& Consumo de tabaco & 2 & 6 & 5.75 & .83 & -3.38 & 10.66 \\
& Consumo de alcohol & 2 & 6 & 5.11 & 1.26 & -0.96 & -0.54 \\
& Información sobre diabetes & 2 & 6 & 4.49 & 1,09 & -0.31 & -0.58 \\
Red de apoyo & Red de apoyo social & 4 & 12 & 10.81 & 1.58 & -1.64 & 3.04 \\
social & & 6 & 29 & 15.87 & 4.39 & 0.31 & -0.18 \\
Estrategias de & Búsqueda de apoyo social & 10 & 40 & 27.23 & 6.57 & -0.17 & -0.73 \\
aproximación & Pensamiento positivo & 5 & 25 & 18.87 & 4.62 & -0.59 & -0.31 \\
& Plany búsqueda de soluciones & 7 & 25 & 18.30 & 4.49 & -0.32 & -0.62 \\
Estrategias & Evitación & 7 & 35 & 18.76 & 5.83 & 0.23 & -0.52 \\
evitación & Pensamiento desiderativo & 5 & 25 & 17.37 & 5.78 & -0.39 & -0.82 \\
& Resignación & 5 & 25 & 14.65 & 4.23 & 0.20 & -0.16 \\
& Culpar a otros & 6 & 23 & 13.71 & 3.57 & -0.10 & -0.15 \\
\hline
\end{tabular}


Como se puede evidenciar, la muestra exhibe pocos síntomas depresivos, donde los mayores síntomas reportados son fisiológicos, seguido de los motores y, por último, los cognitivos. Sin embargo, al observar la asimetría y la alta variación de los datos se puede señalar que hay personas con moderada y alta depresión.

Hay una alta adherencia al tratamiento en los participantes, donde son las dimensiones consumo de tabaco, consumo de alcohol y adherencia terapéutica las que poseen los puntajes más altos. Sin embargo, observando la asimetría y la variación de los datos se puede señalar que solamente en las dimensiones de "actividad física" e "información sobre diabetes" la distribución se comporta de manera más homogénea.

Existe una moderada-baja red de apoyo social en los participantes con una heterogeneidad baja.

Además, existen diversos usos de estrategias de evitación al problema por los participantes, donde las más empleadas son los pensamientos desiderativos, seguido de resignación, evitación y finalmente culpar a otros. Existe baja variabilidad en la distribución de los datos con tendencia a puntajes medios, a excepción de pensamiento desiderativo donde existe mayor variabilidad.

Referente a las estrategias de aproximación, existe un alto empleo, donde son los pensamientos positivos los que más se usan, seguidos de la planificación y la búsqueda de soluciones y, por último, la búsqueda de apoyo social. Hay baja variabilidad en la distribución de los datos con tendencia a puntajes medios-altos.

\section{Modelo de ecuaciones estructurales (MEE)}

En primer lugar, al evaluar los supuestos del MEE, se observa que se cumplen a excepción de la normalidad, dado que todas las variables tienen algún tipo de desviación a la distribución normal, especialmente la dimensión cognitiva de la depresión y las siguientes dimensiones de adherencia al tratamiento: nutrición, consumo de tabaco y adherencia terapéutica (ver tabla 2). Por tal motivo, se empleó como técnica de estimación del modelo la "asintóticamente libre de distribución (AGL)" debido a su insensibilidad a la no normalidad de los datos (Hair et al.,1999).

De esta manera, se presentan luego del procesamiento, los modelos resultantes en las figura 1 y 2.

Modelo A. Adherencia al tratamiento prediciendo depresión al considerar la red de apoyo social, las estrategias de afrontamiento y el sexo (ver figura 1). 
RELACIÓN ENTRE DEPRESIÓN Y ADHERENCIA AL TRATAMIENTO EN DIABETES TIPO 2

\section{Figura 1.}

Modelo A resultante de adherencia al tratamiento sobre depresión al considerar la red de apoyo social, las estrategias de afrontamiento y el sexo.

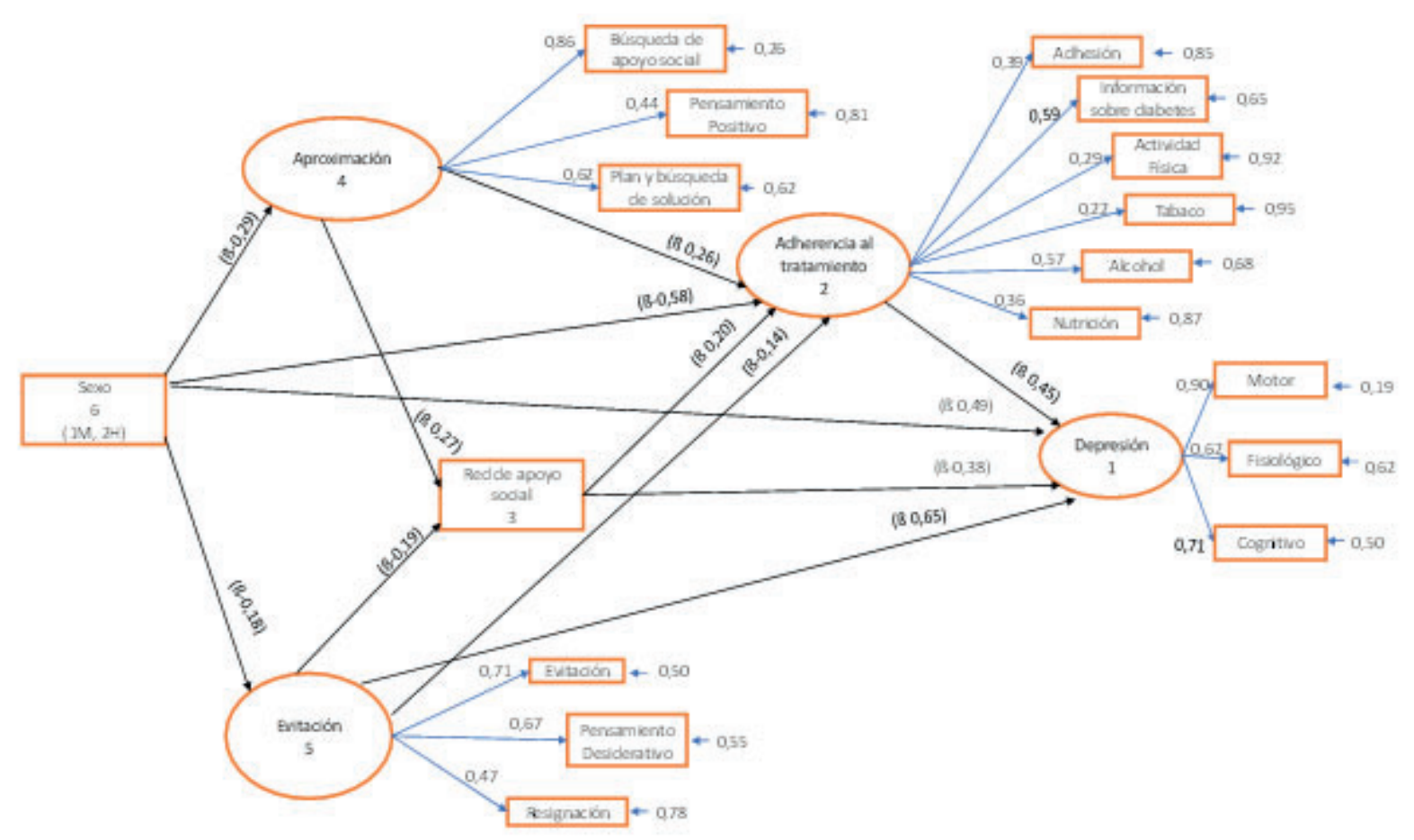

Nota. Líneas negras representan las relaciones de influencias entre las variables (coeficiente Beta). Líneas azules de la variable a la dimensión representan las cargas factoriales de estas últimas sobre su respectivo constructo. Líneas azules pequeñas que apuntan hacia la dimensión representa el error. 
Este modelo obtuvo un $\chi^{2}(123)=484.62, p=.001$, lo cual es significativo. Por tal motivo, da indicio de un ajuste de los datos inadecuado. Sin embargo, Hair et al. (1999) señalan que este estadístico es muy sensible al tamaño de la muestra; por ende, se recomienda interpretarlo en conjunto con las otras medidas de ajuste, las cuales son las siguientes: $\mathrm{GFI}=.97$; RMSEA $=.10$; $\mathrm{AGFI}=.95 ; \mathrm{PNFI}=.51$ у CFI $=.69$.

De acuerdo con Steiger (1990), un RMSEA menor a .10 indica un buen ajuste de los datos. Por su parte, todos los índices de ajuste relacionados con el porcentaje de varianza explicada como el GFI y AGFI deben de exceder de .90 para indicar un buen ajuste a los datos. Referente a los índices de parsimonia tales como el CFI y el PNFI, no existen niveles recomendados de ajuste; sin embargo, cuando se compara entre modelos, se proponen diferencias de .06 a .09 para que se considere como diferencias sustanciales entre ellos (Hair et al., 1999).

Al tomar en consideración de manera general los índices calculados, se afirma que el modelo posee un ajuste aceptable.

El modelo de medida presenta las siguientes características:

Todos los indicadores de la depresión mostraron correlaciones altas con dicha variable. Se concluye que los tres indicadores son medidas fiables de la depresión.

Los indicadores de la adherencia al tratamiento mostraron cargas que oscilaron entre bajas y moderadas. Se concluye que los indicadores actividad física y consumo de tabaco no constituyen medidas fiables de la variable latente. La variable estrategias de aproximación posee correlaciones moderadas y altas con sus indicadores. Se concluye que las tres dimensiones son indicadores fiables de la variable latente estrategias de aproximación.

La variable estrategias evitación tiene correlaciones moderadas y altas con sus indicadores. El indicador culpar a otros se eliminó por no reportar carga significativa con la variable. Se concluye que evitación, pensamiento desiderativo y resignación son medidas fiables de la variable estrategia de evitación.

Al evaluar el modelo de relaciones, se establecen los siguientes resultados:

Se encontró que a mayor adherencia al tratamiento mayor depresión. Por su parte, mayor estrategia de evitación se relaciona con mayor depresión. No se encontró relación con las estrategias de aproximación. Por su parte, a mayor red de apoyo social menor depresión. Por último, se encontró que los hombres fueron los que reportaron mayor depresión.

Con respecto a la adherencia al tratamiento se halló que a mayor red de apoyo social mayor adherencia. Mientras que a mayor empleo de estrategias de aproximación y a menor uso de estrategias de evitación mayor adherencia al tratamiento. Finalmente, se encontró que son los hombres los que menos se adhieren al tratamiento.

Para la red de apoyo social, se obtuvo que a mayor empleo de estrategias de aproximación, menor uso de estrategias de evitación. No se halló relación entre el sexo y la red de apoyo social. En relación con estrategias de afrontamiento, se encontró que las mujeres emplean mayormente tanto las estrategias de aproximación como las estrategias de evitación. 
RELACIÓN ENTRE DEPRESIÓN Y ADHERENCIA AL TRATAMIENTO EN DIABETES TIPO 2

En conclusión, el Modelo "A" posee un ajuste adecuado a los datos, donde las relaciones planteadas fueron verificadas parcialmente.

Modelo B. Depresión prediciendo adherencia al tratamiento, considerando la red de apoyo social, las estrategias de afrontamiento y el sexo (ver figura 2).

Figura 2.

Modelo B resultante de depresión sobre adherencia al tratamiento al considerar la red de apoyo social, las estrategias de afrontamiento y el sexo

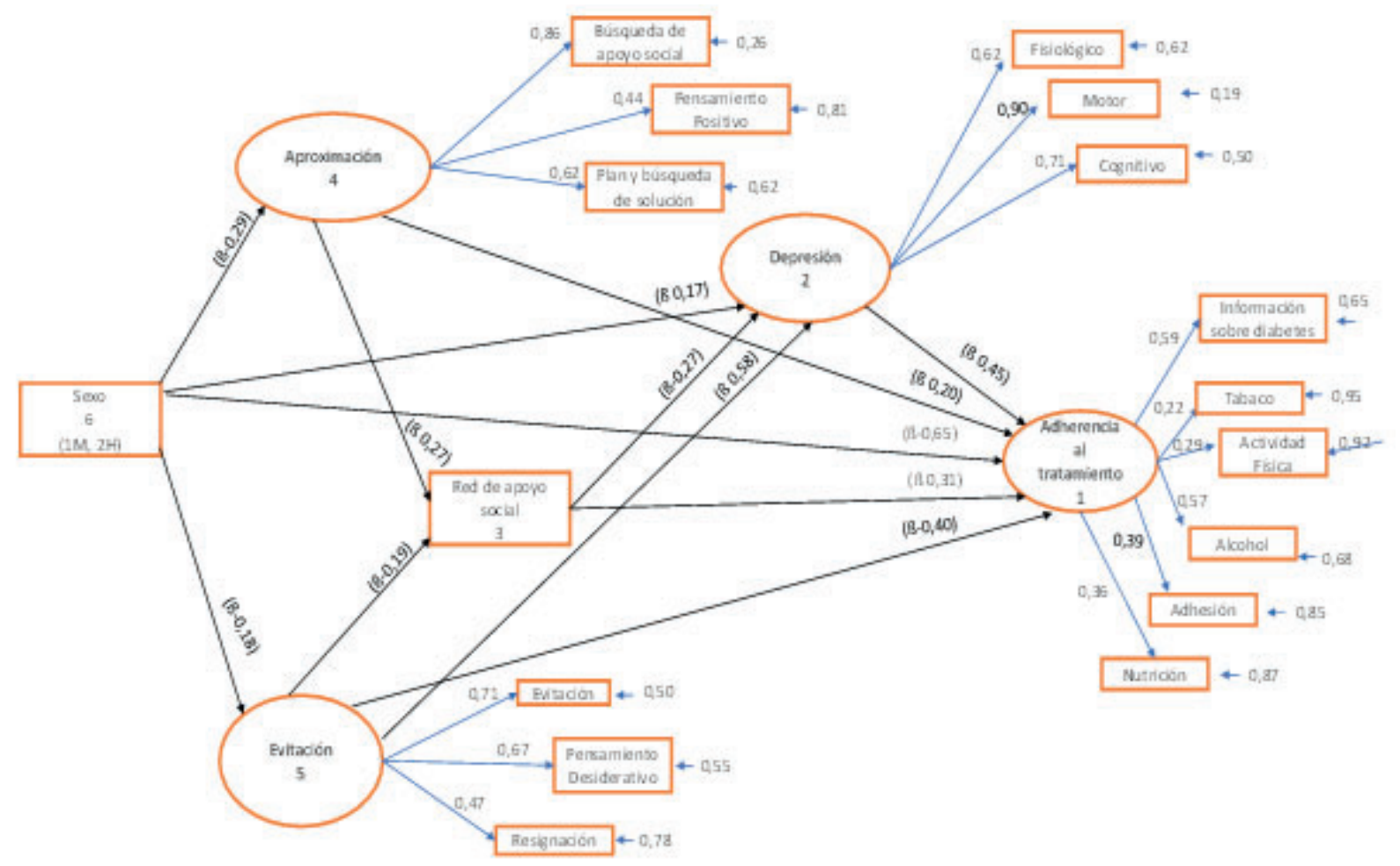

Nota. Líneas negras representan las relaciones de influencias entre las variables (coeficiente Beta). Líneas azules de la variable a la dimensión representan las cargas factoriales de estas últimas sobre su respectivo constructo. Líneas azules pequeñas que apuntan hacia la dimensión representa el error. 
Obtuvo exactamente las mismas medidas de bondad de ajuste del modelo "A": $\chi^{2}(123)=484.62$, $p=.000 ; \mathrm{GFI}=.97 ; \mathrm{RMSEA}=.10 ; \mathrm{AGFI}=.95 ; \mathrm{PNFI}=.51$ y CFI $=.69$.

En cuanto al modelo de relaciones, se hallaron ligeras variaciones en sus magnitudes con respecto al Modelo A, a excepción de las siguientes relaciones que tuvieron una diferencia de mínimo 0.10 puntos:

La relación entre la red de apoyo social y la adherencia al tratamiento se incrementó a .31 (modelo A: .20). Ahora, se trata de una relación igualmente positiva, pero moderada.

La relación entre las estrategias de evitación y la adherencia al tratamiento se incrementó a -.40 (modelo A: -.14). Ahora, es una relación igualmente negativa, pero moderada.

La relación entre sexo y depresión disminuyó a .17 (modelo A: .49). Ahora, consiste en una relación igualmente positiva, pero baja.

La relación entre la red de apoyo social y la depresión disminuyó a -.27 (modelo A: -.38). Ahora, es una relación igualmente negativa, pero baja.

En conclusión, el modelo "B" posee un ajuste adecuado a los datos al tener las mismas características en cuanto a la naturaleza de sus relaciones que el modelo "A".

\section{Discusión}

Las personas con diabetes tienen alta probabilidad de presentar depresión (Caicedo et al, 2012; Domínguez \& Víamonte, 2014; Dahai et al., 2017). En este sentido, Castillo y Aguilar (2011) sostienen que la depresión representa una barrera terapéutica para un adecuado control metabólico, pero se puede considerar que la depresión es el resultado del impacto del tratamiento; es decir, por el cambio drástico de hábitos de salud que este implica.

Derivado a la relación bidireccional entre adherencia y depresión, la presente investigación pretendió determinar el modelo en diabetes que presenta un mayor ajuste de los datos, según la depresión predice o no a la adherencia al tratamiento, al considerar la red de apoyo social, las estrategias de afrontamiento y el sexo como factores influyentes.

Los hallazgos de la investigación muestran que ambos modelos propuestos obtuvieron exactamente el mismo ajuste de los datos a lo planteado; por lo consiguiente, el modelo de medida reportó las mismas cargas y las relaciones encontradas fueron las mismas, aunque con ligeras variaciones en sus magnitudes, donde no existe una ventaja clara de un modelo sobre el otro en cuanto a su poder explicativo.

Las hipótesis sobre la depresión fueron verificadas parcialmente, puesto que se encontró contrario a lo que se esperaba, a mayor adherencia al tratamiento mayor depresión. Por su parte, mayor estrategias de evitación, se relaciona con mayor depresión, tal como se había planteado en las hipótesis; no se encontró relación con las estrategias de aproximación, contrario a lo que se esperaba. La relación planteada de mayor red de apoyo social, menos depresión fue corroborada. Por último, se encontró que los hombres fueron los que reportaron mayor depresión, contrario a lo planteado como hipótesis.

Con respecto a la relación depresión-adherencia al tratamiento, algunos autores afirman que mayor adherencia al tratamiento mayor probabilidad de desarrollar síntomas de depresión (Castillo-Quan et al., 
RELACIÓN ENTRE DEPRESIÓN Y ADHERENCIA AL TRATAMIENTO EN DIABETES TIPO 2

2010; Colunga et al., 2008; Franciosi et al., 2001; Frojan \& Rubio, 2004; Massano-Cardoso et al., 2020), mientras que otros consideran que la relación es opuesta; es decir, menor adherencia mayor probabilidad de desarrollar depresión (Benítez et al., 2011; Escobedo et al., 2007; Wu et al., 2020).

En el modelo A el hecho de que mayor adherencia al tratamiento conlleve a mayor depresión, aunque es contrario a lo que se planteaba como hipótesis, se puede deber a como se sostiene en algunas investigaciones, que el seguimiento del tratamiento en diabetes por su complejidad puede ser más aversivo para el paciente que la posibilidad de desarrollar futuras complicaciones en la condición. El adherirse al tratamiento, sin una buena orientación psicológica, puede conllevar a un cambio brusco del estilo de vida que puede crear reacciones negativas en el paciente, entre las cuales está la depresión.

Por ejemplo, autores como Hoyos et al. (2011), Jacobson-Dickman y Levitsky (2005) y Lerman (2009) sostienen que el empleo de la insulina como tratamiento farmacológico causa una serie de respuestas en la persona con diabetes, que pudieran estar asociadas a la depresión, puesto que algunos pacientes presentan la expectativa de que inyectarse insulina resultará sumamente complicado y puede restringir sus actividades y limitarlos en sus vidas personales. Así, este hallazgo debe interpretarse con cautela, porque no se pretende sostener que la adherencia al tratamiento per se conlleve a síntomas depresivos, sino la forma de cómo se implemente dicha adherencia por parte de la persona con diabetes y del manejo del profesional de salud.

Si se interpreta desde el modelo B, sería una alta depresión la que conllevaría a mayor adherencia al tratamiento. Esto puede explicarse tomando en cuenta lo señalado por Massano-Cardoso et al. (2020) en cuanto a que esta relación negativa encontrada especialmente en la diabetes tipo 2, se pudiera deber a que generalmente se confunden los síntomas de la depresión, con las consecuencias propias de la condición (insomnio, disminución de la libido, decaimiento) y que obviamente llevarían a la persona a tratar de adherirse más al tratamiento para eliminar dichos síntomas. Desde las diferentes explicaciones queda claro que el resultado incita a seguir investigando en la relación adherencia-depresión, en todas sus aristas.

Por su parte, un mayor empleo de estrategias de evitación conlleva a una mayor depresión. Esto concuerda con lo planteado en el área (Bail \& Azzollini, 2012; Parildar et al., 2015; Peralta, 2007; Roger et al., 2005). Tal como afirma Castro-Espinoza et al. (2015), la adaptación a la Diabetes mellitus requiere un proceso mediante el cual las personas afronten, acepten y se ajusten afectivamente a la condición con habilidad y apoyo social; por lo que estrategias como el uso de pensamientos desiderativos, resignación, evitación y el culpar a otros no son cónsonos con este proceso de adaptación y, por ende, podría conllevar a mayores alteraciones emocionales como lo es la depresión. Por su parte, Morrison y Bennett (2008) plantean que generalmente un uso prolongado de estrategias de evitación al problema se corresponde con una menor salud física y mental.

Las estrategias de aproximación no mostraron algún efecto directo sobre la depresión, contrario a lo esperado (Bail \& Azzollini, 2012; Burns et al., 2016), pero esto pudiera sostener la afirmación de Kraaij y Gamefski (2015) que expresa que el estilo de afrontamiento activo o conductual no se encuentra relacionado con la sintomatología depresiva, solo las estrategias de afrontamiento de estilo cognoscitivas como la reevaluación positiva y el ajuste a metas, son las que inciden de forma negativa en el reporte 
de depresión. En este sentido, en la presente investigación, el hecho de no encontrarse relación se pudiera deber a que solo una de las tres dimensiones que constituían las estrategias de aproximación era propiamente de índole cognoscitiva como es "pensamiento positivo".

Se recomienda seguir investigando este hallazgo puesto que el mismo pone en evidencia la relevancia de un determinado tipo de estrategia como son las de evitación o específicamente las de índoles cognoscitivas para el desarrollo de la depresión.

La red de apoyo social mostró un efecto sobre depresión tal como revelan investigaciones anteriores (Gallo et al., 2015; Gomes et al., 2014; Rosland et al., 2014). El apoyo social continúa siendo un factor protector en el área de la diabetes, puesto que el apoyo recibido generalmente permite aumentar la creencia y la autoconfianza de tener habilidad para manejar la condición (Beverly et al., 2021; Teherán et al., 2017); por ende, se pudiera inferir que existirá menos probabilidad de desarrollar depresión.

Para finalizar, se encontró que son los hombres los que reportan mayor nivel de depresión, resultados opuestos a los generalmente encontrados en el área (Álvarez et al., 2014; Benítez et al, 2011; Gohil et al., 2017; Parildar et al., 2015; Salcedo-Rocha et al., 2008; Rivas-Acuña et al, 2011). Sin embargo, al realizar las correlaciones se evidencia que la relación del sexo con las tres dimensiones de la depresión es de acuerdo con lo esperado, es decir, las mujeres son las que reportan mayores síntomas de depresión (Parildar et al., 2015). Se infiere que existe, al menos, una variable supresora en el modelo de medida de la variable latente depresión que hace que la relación se invierta. Se recomienda en próximos estudios identificar estadísticamente las posibles variables supresoras de la relación sexo-depresión en pacientes con diabetes tipo 2.

Las hipótesis relacionadas con la adherencia al tratamiento también fueron verificadas parcialmente, debido a que se halló tal como se esperaba, mayor red de apoyo social se relaciona con mayor adherencia. (Alcaíno et al., 2014; Beverly et al., 2021). Mientras que mayor empleo de estrategias de aproximación, menor uso de estrategias de evitación, se relaciona con mayor adherencia al tratamiento, tal como se estableció como hipótesis. Finalmente, se encontró que son los hombres los que menos se adhieren al tratamiento, lo cual es contrario a lo que se esperaba. Sobre la adherencia al tratamiento, se halló que poseer mayor red de apoyo social, el mayor empleo de estrategias de aproximación, el menor uso de estrategias de evitación y el ser mujer conlleva a mayor adherencia al tratamiento.

El efecto encontrado de la red de apoyo social sobre la adherencia al tratamiento es avalado por investigaciones anteriores en el área (Arteaga et al., 2017; Gallo et al., 2015; Ramkisson et al., 2017); en este sentido, el apoyo social continúa por destacarse como un factor que incrementa la adherencia al tratamiento. Según Shao et al. (2017), una posible explicación consiste en que frecuentemente las personas con un alto apoyo social, también tienen una alta autoeficacia, lo que le permite alcanzar una adecuada adherencia al tratamiento, por lo que se sugiere investigar en próximos estudios el rol de la autoeficacia en la relación apoyo y la adherencia al tratamiento.

Por su parte, los efectos hallados de las estrategias de afrontamiento tanto evitativas como aproximativas sobre la adherencia son cónsonos con lo que se ha encontrado previamente en el área (Bail \& Azzollini, 2012); es decir, a mayor uso de estrategias de aproximación y menos empleo de estrategias de evitación, mayor es la adherencia al tratamiento. Tal como plantea Rondón y Lugli (2013), se necesita 
RELACIÓN ENTRE DEPRESIÓN Y ADHERENCIA AL TRATAMIENTO EN DIABETES TIPO 2

un rol activo por parte de la persona con diabetes para lograr un mayor ajuste a su condición por lo que el uso de pensamientos positivos, la búsqueda de apoyo social y el hacer planes para la búsqueda de soluciones son estrategias adecuadas para lograr un adecuado control, mientras que el empleo de pensamientos desiderativos, evitación y resignación constituyen estrategias que fomentan un inadecuado control de la condición, pues implican un rol pasivo.

Se halló también que las mujeres tienen mayor adherencia al tratamiento, lo cual es contrario a algunas investigaciones (Gohil et al., 2017; Parildar et al., 2015); sin embargo, existen otros estudios que refieren que son las mujeres las que presentan mayor adherencia (Oliveira \& Trujillo, 2017; Ramos et al., 2017); se pudiera inferir que las mujeres tienen mayor disposición a visitar los centros de salud y, por ende, son las más interesadas en mantener su condición controlada, lo que pareciera relacionarse con las teorías de control conductual y locus de control de salud, por lo que se recomienda continuar investigando este aspecto.

Las hipótesis sobre la red de apoyo social también fueron verificadas parcialmente. Mayor empleo de estrategias de aproximación, menor uso de estrategias de evitación, se relaciona con mayor red de apoyo social, tal como se estableció como hipótesis. No se halló relación entre el sexo y la red de apoyo social, contrario a lo que se planteaba.

Sobre la red de apoyo social, el modelo evidenció que el mayor empleo de estrategias de aproximación, y un menor uso de estrategias de evitación, se relaciona con mayor red de apoyo social. No se encontró relación directa con el sexo, pero sí mediada por los dos tipos de estrategias.

Los efectos encontrados de las estrategias de afrontamiento tanto evitativas como aproximativas sobre la red de apoyo social; es decir, mayor empleo de estrategias de aproximación y un menor uso de estrategias de evitación conlleva a una mayor red de apoyo social, se corresponde con lo que se ha encontrado anteriormente en otras investigaciones (Alcaíno et al., 2014; Rondón y Lugli, 2013). Se debe recordar que un tipo de estrategia aproximativa es la "búsqueda de apoyo social", mientras que las conductas "evitativas" y las relacionadas a "culpar a otros" son típicas estrategias de evitación al problema, por lo que es razonable concluir que estas últimas merman la red de apoyo social, en comparación con las estrategias aproximativas que la incrementa.

Con respecto al efecto directo del sexo sobre la red de apoyo social, Rondón y Lugli (2013) afirman que el sexo afecta a la red de apoyo social a través del optimismo y los distintos tipos de estrategias. Hallazgos similares se encontraron en esta investigación, puesto que el sexo ejerce un efecto indirecto sobre la red de apoyo a través de dos vías: a)las mujeres con mayor empleo de estrategias de aproximación tienen mayor red de apoyo social y b)las mujeres con mayor empleo de estrategias de evitación poseen menor red de apoyo social. Los anteriores resultados pudieran sugerir que más que el hecho biológico de ser hombre o mujer son las conductas de estos las que conllevan a poseer una determinada red de apoyo social, por lo que se consideraría que es más un asunto de características conductuales llevadas a cabo por cada sexo, que por el sexo per se.

En relación con las hipótesis relacionadas con las estrategias de afrontamiento, se encontró que las mujeres son las que emplean mayormente tanto las estrategias de aproximación como las estrategias de evitación. Se destaca que se esperaba lo contrario en la relación del sexo con las estrategias de aproximación. 
Así son las mujeres son las que emplean mayormente tanto las estrategias de aproximación como las estrategias de evitación al problema. En este sentido, se evidencia el planteamiento de Roth y Cohen (1986) que establece que ambos tipos de estrategias no se excluyen entre sí y pueden serusadas de forma combinada.

El hecho de que sean las mujeres las que presenten mayor uso de estrategias de evitación es consistente con lo anteriormente encontrado en el área (Gafyels y Wandell, 2005; Parildar et al., 2015; Salcedo-Rocha et al., 2008), puesto que se considera a la mujer más emocional y pasiva en comparación con el hombre. Sin embargo, el que sea la mujer la que también haya reportado mayor empleo de estrategias aproximativas, parece apuntar a que la explicación anterior basada en el estereotipo de mujer como pasiva y emocional no es totalmente correcta. Tal vez, sea la mujer la que utilice cualquier estrategia para solucionar los problemas independientemente de su posición social o de género.

Finalizado el análisis de las relaciones de predicción en el modelo "A" y "B", se puede afirmar que la mayoría de las hipótesis planteadas fueron verificadas.

En cuanto al nivel de medida de las variables estudiadas en ambos modelos, solamente el correspondiente al de "adherencia al tratamiento" es el que refleja tener indicadores que no son medidas válidas, puesto que sus dimensiones "actividad física" y "consumo de tabaco" reportan cargas factoriales, aunque significativas estadísticamente, muy bajas, por lo que existe en esta variable un problema de validez.

Sobre la dimensión "actividad física", se destaca que en la validación original del instrumento realizada por López et al. (2003) y en la adaptación venezolana realizada por Rondón et al. (2014) es la dimensión que reporta la menor consistencia interna y la que explica menos varianza, por lo que en consecuencia se recomienda revisar el contenido de estos ítems, puesto que parece no medir adecuadamente la dimensión, especialmente al ser esta uno de los pilares de las conductas que deben realizar la población con diabetes para mantener controlada su condición.

Sobre la dimensión "consumo de tabaco", se resalta que estuvo constituida solo por dos ítems los cuales no mostraron mayor varianza debido a que el $91 \%$ de la muestra refirió no fumar. Al respecto, se expresa que esa falta de variabilidad pudo haber sido la razón por la cual esta dimensión no tuviera una carga alta con la variable. En este sentido, Anastasi y Urbina (1998) refieren que las diferencias individuales (variabilidad) influyen en cualquier coeficiente de correlación, por lo que sí existe una característica muy homogénea en una determinada muestra, que resultaría imposible de usar para predecir otra característica en dicha muestra.

Además, en la variable "estrategias de evitación" se eliminó el indicador "culpar a otros" por no poseer una carga significativa. Esta dimensión es la única que posee ítems invertidos; es decir, un mayor puntaje en estos ítems indica que la persona asume por completo la causa de su condición. En este sentido, se pudiera plantear en futuros estudios investigar si realmente el "culpar a otros" es una estrategia de evitación al problema, puesto que en esta, tal como se mide en este cuestionario, la persona no niega que el problema haya sucedido. Solamente niega que es el responsable total de que su salud se encuentre afectada, por lo que esta posición se considera adecuada y no necesariamente de evitación, porque de acuerdo con Wallston (2004), las personas que más conservadas tienen su salud consideran que estar saludable es una responsabilidad compartida; es decir, depende de sus propias acciones como de las acciones de los médicos o especialistas tratantes. 
RELACIÓN ENTRE DEPRESIÓN Y ADHERENCIA AL TRATAMIENTO EN DIABETES TIPO 2

Se puede concluir que ambos modelos en diabetes (adherencia al tratamiento- depresión versus depresión- adherencia al tratamiento) presentan la misma bondad de ajuste por lo que por el empleo del Modelo de Ecuaciones Estructurales es igual que la adherencia al tratamiento prediga a la depresión o que esta última sea la que prediga la adherencia al tratamiento.

Una de las limitaciones del presente estudio fue el empleo de un diseño transeccional; por ende, se recomienda replicar el estudio al emplear un diseño longitudinal de panel con el objetivo de verificar la relación causal entre adherencia al tratamiento y la depresión. La muestra estudiada reportó bajo nivel de depresión y alto de adherencia al tratamiento, por lo que los resultados de la predicción no consideran todos los niveles de ambas variables y se constituye en una limitante. Se recomienda seleccionar muestras con mayor equivalencia en la diversidad de los puntajes de ambas escalas.

De igual manera, se deben tomar en cuenta las limitaciones provenientes de la conceptualización y medición de las variables, porque dependiendo de cómo se defina y se midan las variables pueden variar la interpretación; por ejemplo, no es igual trabajar redes sociales como el número y la calidad de personas o como la percepción de apoyo de estas redes. Además, se debe destacar que para el presente estudio se emplearon escalas validadas en trabajos de grados. En ese sentido, son instrumentos no estandarizados y que no presentan puntos de corte, por lo que se recomienda tomar con cautela los resultados encontrados al momento de su interpretación en otras poblaciones.

Tomando en consideración las limitaciones, los resultados pueden servir de base para el diseño de intervenciones en el área de adherencia al tratamiento; por ejemplo, se podría entrenar a las personas en el manejo de emociones negativas y en la forma de afrontar a las situaciones estresantes al enfatizar en poblaciones de riesgo. Dado que el poseer red de apoyo social y menor estrategia de evitación constituyen variables protectoras en la adherencia al tratamiento, se recomienda a los profesionales de la salud considerar el manejo de las mencionadas variables. Por último, con las variables de estudio, se podría crear un perfil de las personas con diabetes con mayor probabilidad de desarrollar depresión. Así, se identificaría rápidamente a esta población vulnerable para posteriormente su intervención psicoterapéutica.

\section{Referencias}

Alcaíno, D., Bastías, N., Benavides, C., Figueroa, D., \& Luengo, C. (2014). Cumplimiento del tratamiento farmacológico de la diabetes mellitus de tipo 2 en adultos mayores: influencia de los factores familiares. Gerokomos, 25, 9-12. https://www.researchgate.net/deref/http $\% 3 \mathrm{~A} \% 2 \mathrm{~F} \% 2 \mathrm{Fdx}$.doi. org\%2F10.4321\%2FS1134-928X2014000100003

Anastasi, A., \& Urbina, S. (1998). Tests psicológicos. Prentice Hall.

Álvarez, C., Sánchez, Y., Bombín, R., Arquellada, M., \& Guerrero, A. (2014). Control de la diabetes, algo más que una cifra de hemoglobina glicosilada. Enfermería en Cardiología, 62, 43-48. https://www. enfermeriaencardiologia.com/wp-content/uploads/62 05.pdf

Arteaga, A., Cogollo, R., \& Muñoz, D. (2017). Apoyo social y control metabólico en la diabetes mellitus tipo 2. Revista CUIDARTE, 8(2), 1668-1676. http://dx.doi.org/10.15649/cuidarte.v8i2.405.

Bail, V., \& Azzollini, S. (2012). Actitudes, afrontamiento y autocuidado en pacientes con diabetes tipo 2. Revista Argentina de Salud pública, 3(10), 15-23. http://rasp.msal.gov.ar/rasp/articulos/volumen10/Pupko.pdf 
Benítez, A., Gonzales, L., Bueno, E., Agüero, F., Alsina, S., Melgarejo, M., Ramírez, A., \& Vera-Ruff-Inelli, J. (2011). Depresión y diabetes. Revista de la ALAD, 1(4), 162-169.

Beverly, E., Ritholz, M., \& Dhanyamraju, K. (2021). The buffering effect of social support on diabetes distress and depressive symptoms in adults with Type 1 and Type 2 diabetes. Research: educational and psychological aspects. 38(4), 1-10. https://doi.org/10.1111/dme.14472

Burns, R., Deschênes, S., \& Schmitz, N. (2016). Associations between coping strategies and mental health in individuals with type 2 diabetes: Prospective analyses. Health Psychology, 35(1), 78-86. DOI: 10.1037/ hea 0000250 .

Caicedo, D., Duarte, C., González, k., Gualdrón, E., Guamán, R., Igua, D., \& Infante, V. (2012). Factores no farmacológicos asociados al control de la diabetes mellitus tipo 2. Médicas UIS, 25, 29-43. DOI: 10.18273/revmed

Cardozo, I., Guarino, L., \& Rondón, J. (2016). Validación del Cuestionario Tridimensional para la Depresión (CTD) en población venezolana con enfermedades crónicas. Revista de Psicopatología y Psicología Clínica, 21(3), 191-200. DOI: 10.5944/rppc.vol.21.num.3.2016.17812

Castillo, Y., \& Aguilar, C. (2011). Depresión, control glucémico y diabetes. Revista de la Asociación Latinoamericana de Diabetes, 1, 141-142.

Castillo-Quan, J., Barrera-Buenfil, D., Pérez-Osorio, J., \& Álvarez-Cervera, F. (2010). Depresión y diabetes: de la epidemiología a la neurobiología. Revista de Neurología, 51(6), 347-359. https://doi.org/10.33588/ rn.5106.2009398

Castro-Espinoza, J., Gallegos-Cabriales, E., \& Frederickson, K. (2015). Análisis evolutivo del concepto de adaptación a la diabetes tipo 2. Aquichan, 15(1), 52-59. DOI: 10.5294/aqui.2015.15.1.6

Colunga, C., García de Alba, J., Salazar, J., \& Ángel, M. (2008). Diabetes tipo 2 y depresión en Guadalajara, México. 2005. Revista de Salud Pública, 10(1), 137-149. https://doi.org/10.1590/S0124-00642008000100013

Congreso de la República de Venezuela. (1978). Ley de ejercicio de la Psicología. Aprobado en Gaceta Oficial de la República de Venezuela N².306 Extraordinario.

Dahai, P., Balamurugan, G. \& Basavaraia, A. (2017). Prevalence and predictors of depression among diabetes mellitus in adult population. Journal of Psychiatry, 20(6), 430-433. doi:10.4172/2378-5756.1000430

De Groot, M., Crik, K., Long, M, Saha, Ch., \& Shubrook, J. (2016). Lifetime duration of depressive disorders in patients with type 2 diabetes. Diabetes Care, 39, 2147-2181. DOI: 10.2337/dc16-1145. Epub 2016 Oct 11

Domínguez, M., \& Víamonte, Y. (2014). Ansiedad, depresión y vulnerabilidad al estrés ante el diagnóstico reciente de diabetes mellitus tipo 2. Gaceta Médica Espirituana, 16, 1-9. http://scielo.sld.cu/pdf/gme/v16n3/gme09314.pdf

Escobedo, C., Díaz, N., Lozano, J., Rubio, A., \& Valera, G. (2007). Descontrol metabólico relacionado con depresión según el inventario de Beck en pacientes diabéticos tipo 2 de reciente diagnóstico. Medigraphic Artemisa. 23(5), 385-90. https://www.medigraphic.com/pdfs/medintmex/mim-2007/mim075e.pdf

Franciosi, M., Pellegrini, F., DeBernardis, G., Belfiflio, M., Cavaliere, D., Di Nardo, B., Greenfield, S., Kaplan, S., Sacco, M., Tognoni, G., Valentini, M., \& Nicolucci,A. (2001). Impact of blood glucose self-monitoring on metabolic control and quality of life in type 2 diabetic patients. Diabetes Care, 24, 1870-1877. https://doi.org/10.2337/diacare.24.11.1870 
RELACIÓN ENTRE DEPRESIÓN Y ADHERENCIA AL TRATAMIENTO EN DIABETES TIPO 2

Feldman, L. (2002). Roles sociales, factores psicosociales y salud en mujeres trabajadoras. [Trabajo de ascenso para optar a la categoría de titular]. Universidad Simón Bolívar, Caracas.

Froján, M., \& Rubio, R. (2004). Análisis discriminante de la adhesión al tratamiento en la diabetes mellitus insulinodependiente. Psicothema, 16(4), 548-554. http://www.psicothema.com/pdf/3030.pdf

Gafyels, C. \& Wandell, P. (2005). Coping strategies in men and women with type 2 diabetes in Swedish primary care. Diabetes Research and Clinical Practice, 71(3), 280-289. DOI: 10.1016/j.diabres.2005.07.001.

Gallo, L., Fortmann, A., McCurley, J., Isasi, C., Penedo, F., Daviglus, M., Roesch, S., Talavera, G., Gouskova, N., González, F., Schneiderman, N., \& Carnethon, M. (2015). Associations of structural and functional social support with diabetes prevalence in U.S. Hispanics/Latinos: Results from the HCHS/SOL Sociocultural Ancillary Study. Journal Behavioral Medicine, 38, 160-170. DOI: 10.1007/s10865-014-9588-z

Gohil, E., Charak, R., Rashid, H., \& Sharma, P. (2017). Quality of life and depression among patients with type I diabetes: a study of gender differences. The International Journal of Indian Psychology, 4(2), 90-97. https:// doi.org/18.01.012/20170402

Gomes, E., Pereira, R., \& Cunha, E. (2014). Relationship between socio-demographic, clinical and psychosocial variables in patients with type 2. Analise Psicológica, 1(32), 63-77. https://doi.org/10.14417/ap.720

González, J., Peyrot, M., McCarl, L., Collins, E., Serpa, L., Mimiaga, M., \& Safren, S. (2008). Depression and Diabetes Treatment Nonadherence: A Meta-Analysis. Diabetes Care, 31(12): 2398-2403.

Hair, J., Anderson, R., Tatham, R., \& Black, W. (1999). Análisis multivariante. Prentice Hall.

Holt, R., De Groot, M., \& Hill, S. (2014). Diabetes and depression. Current Diabetes Reports, 14(6), 491-505. DOI: 10.1007/s11892-014-0491-3

Hoyos, T., Arteaga, M., \& Muñoz, M. (2011). Factores de no adherencia al tratamiento en personas con diabetes mellitus tipo 2 en el domicilio. La visión del cuidador familiar. Investigación y Educación en Enfermería, 29, 194-203.

Iglesias, S. \& Sulé, M. (2003). Introducción al análisis multivariante. En J. Lévy. \& J. Varela (Eds.). Análisis multivariante para las ciencias sociales (pp. 1-40). Prentice hall.

Jacobson-Dickman, E., \& Levitsky, L. (2005). Agentes orales en el tratamiento de la diabetes mellitus en niños y adolescentes. Clínicas Pediátricas de Norteamérica, 52, 1689-1703. https://dialnet.unirioja.es/servlet/ articulo? codigo $=2187907$

Jiménez, G. (2002). La depresión desde una perspectiva tridimensional: Elaboración de un instrumento de medida. [Tesis doctoral no publicada]. Universidad Complutense de Madrid.

Jiménez, G., \& Miguel-Tobal, J. (2003). El cuestionario tridimensional para la depresión (ctd): Un nuevo instrumento para medir la tristeza/depresión. Ansiedad y Estrés, 9 (1), 17-34.

Kaltman, S., Serrano, A., Talisman, N., Magee, M., Cabassa, L, Pulgar-Vidal, O., \& Peraza, D. (2016). Type 2 diabetes and depression. A pilot trial of an integrated self-management intervention for latino immigrants. The Diabetes Educator, 42(1), 87-95. DOI: 10.1177/0145721715617536 
Karlsen, B., Oftedal, B., \& Bru, E. (2012). The relationship between clinical indicators, coping styles, perceived support and diabetes-related distress among adults with type 2 diabetes. Journal of Advanced Nursing, 68(2), 391-401. https://doi.org/10.1111/j.1365-2648.2011.05751.x

Kraaij, V., \& Gamefski, N. (2015). Cognitive, behavioral and goal adjustment coping and depressive symptoms in young people with diabetes: A search for intervention targets for coping skills training. Journal of Clinical Psychology in Medical Settings, 22(1), 45-53. https://doi.org/10.1007/s10880-015-9417-8

Lazarus, R. \& Folkman, S. (1986). Estrés y procesos cognitivos. Ediciones Martínez Roca.

Lerman, I. (2009). Barreras que dificultan la aplicación temprana de insulina en el paciente con diabetes tipo 2. Asociación Latinoamericana de Diabetes, 17(2), 66-68. http://www.revistaalad.com/pdfs/0906 BarrDif.pdf

López, J. Arisa, C. Rodríguez, J., \& Munguía, C. (2003). Construcción y validación inicial de un instrumento para medir el estilo de vida en pacientes con diabetes mellitus tipo 2. Salud Pública de México, 45, 259-268. http://www.scielo.org.mx/pdf/spm/v45n4/a04v45n4.pdf

Lyketsos, C. (2010). Depression and diabetes: More on what the relationship might be. The American Journal of Psychiatry, 167(5), 498-500. https://doi.org/10.1176/appi.ajp.2010.10020243

Massano-Cardoso, I., Daniel, F., Rodrigues, V., \& Galhardo, A. (2020). Depressive symptoms in Type 1 and Type 2 Diabetes Mellitus and its relationship with glycemic control. Revista Portuguesa de Investigación Comportamental y social. 6(2), 40-49. https://doi.org/10.31211/rpics.2020.6.1.166

Montiel-Carbajal, M., \& Domínguez-Guedea, M. (2011). Aproximación cualitativa al estudio de la adhesión al tratamiento en adultos mayores con DMT2. Revista Latinoamericana de Medicina Conductual, 1(2), 7-18. https://www.redalyc.org/articulo.oa?id=283021986002

Montero, I., \& León, O. (2007). A guide for naming research studies in Psychology. International Journal of Clinical and Health Psychology, 7(3), 847-862. http://www.aepc.es/ijchp/GNEIP07_es.pdf

Moral de la Rubia, J., \& Cerda, M. (2015). Predictores psicosociales de adherencia a la medicación en pacientes con diabetes tipo 2. Revista Iberoamericana de Psicología y Salud, 6(1), 19-27. https://doi. org/10.1016/S2171-2069(15)70003-7

Morrison, V., \& Bennett, P. (2008). Psicología de la salud. Pearson Educación, S.A.

Oliveira, V., \& Trujillo, S. (2017). Autocuidado y adherencia al tratamiento en pacientes con diabetes mellitus del servicio de medicina interna del hospital universitario Dr. Ángel Larralde. Medicina Interna, 33(1), 24-34. https://www.svmi.web.ve/ojs/index.php/medint/article/view/413/406

Ortiz, M., \& Fernández-Pera, M. (2018). Modelo de Ecuaciones Estructurales: Una guía para ciencias médicas y ciencias de la salud. Terapia Psicológica, 36 (1), 47-53 http://dx.doi.org/10.4067/ $\underline{\mathrm{s} 0718-48082017000300047}$

Otero, A., Zunzunegui, M., Béland, F., Rodríguez, A., \& García, M. (2006). Relaciones sociales y envejecimiento saludable. Fundación BBVA. 
RELACIÓN ENTRE DEPRESIÓN Y ADHERENCIA AL TRATAMIENTO EN DIABETES TIPO 2

Parildar, H., Cigerli, O., \& Guvener, N. (2015). Depression coping strategies, glycemic control and patient compliance in type 2 diabetic patients in an endocrine outpatient clinic. Pakistan Journal of Medical Sciences, 31(1), 19-24. https://doi.org/10.12669/pjms.311.6011

Peralta, G. (2007). Depresión y diabetes. Avances en Diabetología, 23(2), 105-108. http://www.avancesendiabetologia. org/revista/revistaVerArticulo.asp?idRevista $=12 \&$ idArticulo $=140 \& p a=$ seminarios

Pouwer, F., Schram, M.T., Iversen, M.M., \& Holt, R.I.G. (2020). How 25 years of psychosocial research has contributed to a better understanding of the links between depression and diabetes. Diabetic Medicine, 37, 383-392. https://doi.org/10.1111/dme.14227

Rahimi, M., Jalali, M., Nouri, R., \& Rahimi, M. (2020). The mediating role of resilience and diabetes distress in relationship between depression and treatment adherence in Type 2 Diabetes among iranian patients. Journal Community Health Research. 9(2), 107-118.

Ramos, Y., Morejón, R., Gómez, M., Reina, M., Rangel, C., \& Cabrera, Y. (2017). Adherencia terapéutica en pacientes con diabetes mellitus tipo 2. Revista Finlay, 7(2), 89-98. https://www.medigraphic.com/pdfs/ finlay/fi-2017/fil72d.pdf

Ramkisson, S., Pillay, B., \& Sibanda, W. (2017). Social support and coping in adults with type 2 diabetes. African Journal of Primary Health Care \& Family Medicine, 9(1), 1-8. https://doi.org/10.4102/phcfm.v9i1.1405

Reyes, G. (2013). Psicoterapia psicodramática: una forma de diagnóstico y tratamiento para la depresión. Revista Brasileira de Psicodrama, 21(2), 53-64.

Rivas-Acuña, V., García, H. Cruz, A., Morales, F., Enríquez, R., \& Román, J. (2011). Prevalencia de ansiedad y depresión en las personas con diabetes mellitus tipo 2. Salud en Tabasco, 17(1-2), 30-35. https://www. redalyc.org/pdf/487/48721182005.pdf

Rodrígues, E., Egidio, A., \& Cardoso, A. (2014). The role of depression on glycemic control. Journal of Endocrinology Metabolism, 4(5-6), 119-120. http://dx.doi.org/10.14740/jem247w

Rodríguez-Marín, J., Pastor, M., \& López-Roig, S. (1993). Afrontamiento, apoyo social, calidad de vida y enfermedad. Psycothema, 5(1), 349-372. http://www.psicothema.com/psicothema.asp?id=1148

Rogers, M., Hansen, N., Levy, B., Tate, D., \& Sikkema, K. (2005). Optimism and coping with loss in bereaved HIV-infected men and women. Journal of Social and Clinical Psychology, 24(3), 341-360. https://doi. org/10.1521/jscp.24.3.341.65619

Rondón, J. (2018). Relación entre depresión y adherencia al tratamiento en diabetes tipo 2: Modelo explicativos. [Tesis de doctorado no publicada]. Universidad Central de Venezuela.

Rondón, J., \& Lugli, Z. (2010). Influencia del tipo de diabetes, estrategias de afrontamiento, sexo y optimismo sobre el apoyo social. [Tesis de Maestría no publicada]. Universidad Simón Bolívar, Caracas.

Rondón, J., \& Lugli, Z. (2013). Efectos del tipo de diabetes, estrategias de afrontamiento, sexo y optimismo en el apoyo social. Revista de Psicopatología y Psicología Clínica, 18(3), 193-207. https://www.aepcp.net/ arc/03 2013 n3 rondon lugli.pdf 
Rosland, A., Piette, J., Lyles, C., Parker, M., Moffet, H., Adler, N., Schillinger, D., \& Karter, A. (2014). Social support and lifestyle vs. medical diabetes self-management in the diabetes study of Northern California (Distance). Annals of Behavioral Medicine, 48(3), 438-447.https://doi.org/10.1007/s12160-014-9623-X

Roth, S., \& Cohen, L. (1986). Approach, avoidance, and coping with stress. American Psychologist, 41(7), 813819. https://doi.org/10.1037/0003-066X.41.7.813

Salcedo-Rocha, A., García de Alba-García, J., Frayré-Torres, M., \& López-Coutino, B. (2008). Género y control de diabetes mellitus 2 en pacientes del primer nivel de atención. Revista Médica del Instituto Mexicano de Seguro Social, 46(1), 73-81. https://www.medigraphic.com/pdfs/imss/im-2008/im0811.pdf

Serrano, C., Zamora, K., Navarro, M., \& Villarreal, E. (2012). Comorbilidad entre depresión y diabetes mellitus. Medicina Interna de México, 28(4), 325-328. https://www.medigraphic.com/pdfs/medintmex/mim-2012/ $\underline{\text { mim124d.pdf }}$

Shao, Y., Liang, L., Shi, L., Wan, Ch., \& Yu, S. (2017). The effect of social support on glycemic control in patients with type 2 diabetes mellitus: the mediating roles of self-efficacy and adherence. Journal of Diabetes Research, 2017, 1-8. https://doi.org/10.1155/2017/2804178

Shrestha, M., Ng, A., Al-Ghareeb, A., Alenazi, F., \& Gray, R. (2020). Association between subthreshold depression and self-care behaviors in people with type 2 diabetes: a systematic review of observational studies. Systematic Reviews, 9, 2-9. https://doi.org/10.1186/s13643-020-01302-z

Steiger, J. (1990). Structural model evaluation and modification: An interval estimation approach. Multivariate Behavioral Research, 25(2), 173-180. http://www.ww.w.statpower.net/Steiger\%20Biblio/Steiger90b.pdf

Stolcke, V. (2000). ¿Es el sexo para el género lo que la raza para la etnicidad... y la naturaleza para la sociedad? [versión electrónica], Política y Cultura, 25-60.

Teherán, A., Mejía, M., Álvarez, L., Muñoz, Y., Barrera, M., \& Cadavid, V. (2017). Relación entre el apoyo social y las complicaciones agudas de la diabetes tipo 2: un estudio de corte transversal. Revista Ciencias de la Salud, 2(5), 211-222. http://dx.doi.org/10.12804/revistas.urosario.edu.co/revsalud/a.5757

Toro-Tobar, R., Grajales-Giraldo, F., \& Sarmiento-López, J. (2016). Riesgo suicida según la tríada cognitiva negativa, ideación, desesperanza y depresión. Aquichan, 16(4), 473-486.

Vallejo, J. (2011). Introducción a la psicopatología y psiquiatría (7a ed). Elsevier Masson.

Wallston, K. A. (2004). Control and health. En N. Anderson (Ed.) Encyclopedia of Health \& Behavior (pp. $217-$ 220). Sage.

Westby, M., Norman, G., Vedhara, K., Game, F., \& Cullum, N. (2020). Systematic Review or Meta-analysis Psychosocial and behavioural prognostic factors for diabetic foot ulcer development and healing: a systematic review. Diabetic Medicine, 37, 1244-1255. https://doi.org/10.1111/dme.14310

Wu, C.S., Hsu, L.Y., \& Wang, S.H. (2020). Association of depression and diabetes complications and mortality: a population-based cohort study. Epidemiology and Psychiatric Sciences. 29(96), 1-9. https://doi.org/10.1017/ $\underline{\text { S2045796020000049 }}$ 
RELACIÓN ENTRE DEPRESIÓN Y ADHERENCIA AL TRATAMIENTO EN DIABETES TIPO 2

Young-Hyman, D., De Groot, M., Hill-Briggs, F., Gonzalez, J., Hood, K., \& Peyrot, M. (2016). Psychosocial care for people with diabetes: a position statement of the american diabetes association. Diabetes Care, 39(12), 2126-2140. https://doi.org/10.2337/dc16-2053

Zaragoza, J. (2006). Severidad de la EPOC y calidad de vida: El rol moderador del afrontamiento y la autoeficacia. [Tesis de Maestría no publicada]. Universidad Simón Bolívar, Caracas.

Recibido: 15 de setiembre de 2019 Revisión recibida: 23 de abril de 2021

Aceptado: 01 octubre 2021 


\section{Sobre el autor y la autora:}

José Eduardo Rondón Bernard es Doctor en Psicología e investigador docente del Instituto de Psicología de la Universidad Central de Venezuela (UCV) y docente de la Universidad Católica Andrés Bello (UCAB) de Caracas, Venezuela. Su línea de investigación es "variables psicosociales asociadas al proceso salud-enfermedad". Actualmente, es el director del Posgrado en Psicología de la Universidad Católica Andrés Bello (UCAB).

Luisa T. Angelucci Bastidas es doctora en Psicología, profesora jubilada de la Universidad Simón Bolívar (USB) y profesora activa de la Universidad Católica Andrés Bello (UCAB) de Caracas, Venezuela. Funge como directora del Centro de Investigación y Evaluación Institucional de la UCAB. Áreas de interés: Psicología de la salud, Psicología social, Evaluación universitaria, Metodología de la investigación, Psicometría.

Publicado en línea: 28 de diciembre de 2021 